\title{
Long-term prognosis of treated acute massive pulmonary embolism
}

\author{
R. J. C. HALL, G. C. SUTTON, AND I. H. KERR \\ From the Departments of Cardiology and Radiology, Brompton Hospital, Fulham Road, London
}

Seventy-two patients surviving initial treatment by pulmonary embolectomy, streptokinase, or heparin for acute massive pulmonary embolism have been reviewed 1 to 9 years later. Of these patients, 17 had additional cardiorespiratory disease. There were 12 late deaths (41\% of those patients with, and $9 \%$ of those patients without additional cardiorespiratory disease). No death was due to chronic pulmonary hypertension, and none to certain recurrence of pulmonary embolism. The causes of death were malignant disease ( 6 patients), cardiopulmonary disease other than pulmonary embolism (4 patients), and 'sudden' (2 patients). Though venous interruption surgery was not used, and long-term anticoagulation rare, the incidence of possible or probable recurrent embolism was low (6\%). There was no definite evidence of persistence or subsequent development of pulmonary hypertension after treatment in any patient. Resolution of embolism as judged by symptoms, signs, electrocardiogram, and chest x-ray examination was almost always complete. Some pulmonary arteriograms 1 to 6 months after initial treatment showed minor abnormalities, but others at a later date were normal. Perfusion lung scans carried out 1 to 8 years after initial treatment often showed minor abnormalities.

The fate of patients with acute massive pulmonary embolism may be sudden death, deterioration over a matter of hours or days resulting in death, or complete recovery taking a varying period of time. It has been suggested that some patients initially presenting with acute pulmonary embolism may develop chronic pulmonary hypertension (Magidson and Jacobson, 1955; Wood, 1956; Phear, 1960). Speculation exists whether chronic thromboembolic pulmonary hypertension represents the end-result of unresolved massive pulmonary embolism or the long-term accumulation of repeated unresolved minor emboli (Dalen and Alpert, 1975) in untreated patients.

During the period 1965 to 1973,88 patients with acute massive pulmonary embolism were referred to the Brompton Hospital for treatment. Confirmation of the initial diagnosis was made in every case either by pulmonary arteriography or at pulmonary embolectomy. Many of these patients survived the initial episode of pulmonary embolism and form a group in which the long-term prognosis of pulmonary embolism can be assessed. This study was concerned with the causes of late death, the degree of resolution of pulmonary embolism, whether chronic pulmonary hypertension developed, and the incidence of recurrence of embolism.

Received for publication 28 February 1977

\section{Definition of patients}

During 1965 to 1973,88 patients with acute massive pulmonary embolism were treated; of these, 66 (75\%) had massive embolism without pre-existing additional cardiorespiratory disease, while 22 $(25 \%)$ had significant cardiorespiratory disease in addition. 'Massive' embolism was defined as involvement of more than 50 per cent of the major pulmonary arteries and was assessed in 61 patients by pulmonary arteriography (Miller et al., 1971), and at pulmonary embolectomy in 27 patients. All patients were referred for treatment from other hospitals.

Of the 88 patients, 85 had a clinically identifiable acute episode of massive embolism. Of these 85 patients, 35 were seen within 12 hours of the massive event, 45 were seen between 12 and 48 hours, and the remaining 5 were seen between 48 and 96 hours. Some of these 85 patients had episodes suggestive of minor pulmonary embolism with pulmonary infarction before the massive episode, but these never preceded the massive embolism by more than two weeks. The remaining 3 patients, all in the group with additional cardiorespiratory disease, had massive embolism with longer histories and no identifiable acute episode.

Sixteen deaths $(18 \%)$ occurred during the initial admission, mainly as the result of intractable 
haemodynamic disturbance caused by massive embolism. Of the 72 surviving patients, 48 were women and 24 were men with an age range of 19 to 71 years (median 50 years) and form the basis of the subsequent discussion.

Cardiorespiratory disease, other than pulmonary embolism, was present in 17 of the 72 surviving patients. Previous myocardial infarction had occurred in 4, generalised left ventricular disease was present in 2, 1 patient had angina, another mitral stenosis, and a further patient had a large peripheral arteriovenous fistula. Three patients had systemic hypertension. Two patients had carcinoma of the bronchus, and 3 had obstructive airways disease.

The predisposing factors to the development of massive pulmonary embolism included operation (for benign and neoplastic disease) or trauma (46 patients), bed-rest ( 4 patients), oral contraceptives (3 patients), pregnancy ( 2 patients), recent myocardial infarction ( 2 patients), mitral stenosis, carcinoma of bronchus, lymphosarcoma, ulcerative colitis, and nephrotic syndrome (1 patient with each). Ten patients had no obvious predisposing factor.

\section{Treatment}

Treatment of patients with acute massive pulmonary embolism has varied over this 9-year period. In the early years, most patients were treated by pulmonary embolectomy, but later many patients were treated with a thrombolytic agent streptokinase (Kabikinase) ${ }^{\star}$ or with heparin. The results of these treatment regimens have been reported elsewhere (Paneth, 1967; Miller et al., 1971; Tibbutt et al., 1974; Miller et al., 1977). Some patients were initially treated with streptokinase and subsequently by embolectomy because of deterioration, and others initially by heparin and then by streptokinase or embolectomy because of deterioration.

The final treatment used in the 72 surviving patients was embolectomy (33 patients), streptokinase (31 patients), and heparin (8 patients).

The majority of patients leaving hospital were treated with anticoagulants (warfarin) for 3 to 6 months. Thirteen patients were kept on long-term oral anticoagulants.

Operations to interrupt the venous system of the legs or the inferior vena cava were not carried out in any of these surviving patients.

* Kabikinase. KabiVitrum Pharmaceuticals Ltd., Uxbridge Road, London W.5.

\section{Causes of late death}

There were 5 late (after initial hospitalisation) deaths $(9 \%)$ in the 55 patients without other cardiorespiratory disease. Malignant disease (carcinoma of the cervix, carcinoma of the breast, reticulumcell sarcoma, and carcinoma of the rectum) accounted for 4 deaths. The patient who died of carcinoma of the rectum was the only one of these patients known to have malignant disease at the time of massive pulmonary embolism; surgery, trauma, and pregnancy were the identifiable predisposing factors in the other 3 patients who later died of neoplastic disease. One patient in whom the initial predisposing factor for pulmonary embolism was immobility, died suddenly 30 months after initial treatment. Necropsy was not carried out on this patient; the clinical circumstances suggested acute myocardial infarction, but the possibility that death was caused by recurrent massive pulmonary embolism cannot be excluded.

Of the 17 patients with additional cardiorespiratory disease, $7(41 \%)$ died 3 to 69 months after the initial embolism. Two died of carcinoma of the bronchus recognised at the time of pulmonary embolism. Three died of left ventricular failure; these were all patients with known left ventricular disease. One of these patients died 6 months after the initial embolus had been treated, and had both old and new pulmonary infarcts at necropsy. One patient with a long history of obstructive airways disease died of coronary artery disease with asthma and bronchitis contributing to her death. The seventh patient died suddenly 38 months after initial treatment for pulmonary embolism; the cause of death is unknown as a necropsy was not performed.

\section{Scope of follow-up}

Of the initial series of 88 patients, 16 died in hospital and 12 subsequently. Of the remaining 60 patients, 56 were traced and recalled for follow-up a minimum of 1 year after initial treatment. The duration of follow-up in these 56 patients is shown in Fig. 1. Patients who died during the follow-up period and the 4 who could not be traced are not included in Fig. 1. The median follow-up period was 5 years.

The history was taken with particular attention to symptoms of cardiopulmonary insufficiency such as dyspnoea and chest pain and to symptoms of recurrent pulmonary embolism such as pleurisy, haemoptysis, sudden dyspnoea, or collapse. Examination was directed to any clinical features suggestive of pulmonary hypertension, e.g. abnormal jugular venous pulse, praecordial pulsation, 


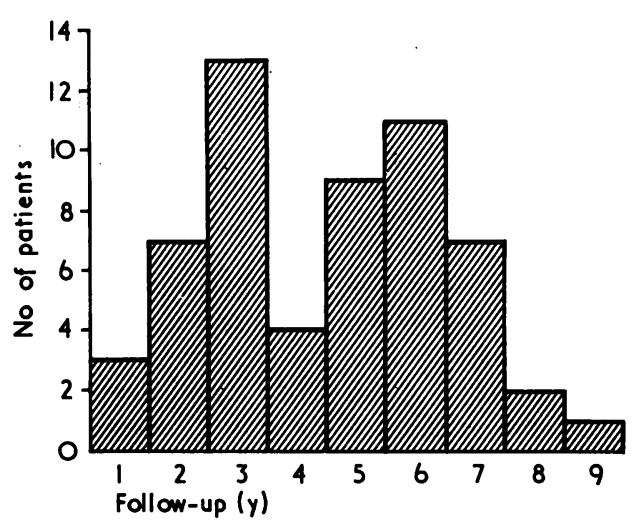

Fig. 1 Duration of follow-up in 56 patients.

or splitting of the second heart sound; to the intensity of the pulmonary component of the second heart sound; and to evidence of other cardiopulmonary abnormalities, e.g. systemic hypertension, left ventricular dysfunction, or obstructive airways disease.

A 12-lead electrocardiogram was taken and compared with the electrocardiogram taken at the time of pulmonary embolism. The plain posteroanterior chest radiograph was analysed for cardiothoracic ratio, size of main pulmonary artery, width of right descending pulmonary artery, areas of oligaemia, height of the diaphragm, linear and other shadows, and the presence of pleural thickening.

Finally, a perfusion lung scan was performed using ${ }^{9 \theta} \mathrm{m}$ Technetium-labelled macroaggregated albumin. Four views were obtained using a 3-inch single crystal detector. Abnormalities recorded were generalised reduction in one lung by comparison with the other, localised areas of underperfusion, and whether these were single or multiple. Quantification of lung scan abnormalities was not attempted, but the lung scan was compared with the chest $x$-ray film after each had been assessed separately.

\section{Results of assessment}

\section{PATIENTS WITHOUT ADDITIONAL}

CARDIORESPIRATORY DISEASE

Of the 46 patients seen whose initial embolism was not complicated by additional cardiopulmonary disease, $31(67 \%)$ were asymptomatic throughout the period of follow-up. Nine patients $(20 \%)$ complained of mild exertional dyspnoea. Three patients had vague chest pains which were not pleuritic, one had an episode of bronchitis during the follow-up period, and one patient had vague chest pains and slight exertional dyspnoea. The only patient who had any history suggestive of recurrence of pulmonary embolism had an episode of pleurisy and haemoptysis 5 years after the initial episode and also complained subsequently of angina. This patient was investigated by cardiac catheterisation 6 years after the initial episode and was shown to have mild pulmonary hypertension (pulmonary artery systolic pressure $40 \mathrm{mmHg}$ ) without pulmonary arteriographic abnormalities, normal left heart pressures, and a normal coronary arteriogram.

Forty-one patients ( $89 \%$ ) had no abnormal physical signs. Mild systemic hypertension was found in 3 patients. There was an ejection systolic murmur at the apex and a double apical cardiac impulse in one patient. One patient had an abnormal second heart sound with abnormally wide expiratory splitting increasing on inspiration: this patient did not have right bundle-branch block, nor was the pulmonary valve closure sound (P2) increased relative to the aortic valve closure sound (A2) on phonocardiography.

The electrocardiogram was normal in 38 patients $(83 \%)$. $\mathrm{T}$ wave inversion in $\mathrm{V} 1$ to $\mathrm{V} 4$ was present in 3 patients, and 2 patients had an $S_{1} Q_{3} T_{3}$ pattern with inverted $T$ waves in V1 to V4. All these abnormalities were also present in the electrocardiogram taken at the time of initial embolism. Complete right bundle-branch block was present in 2 patients: in one this was not initially present. One patient had an rsr $^{1}$ pattern in V1 present initially and at follow-up.

Perfusion lung scans were performed in 39 patients $(85 \%)$ at the time of follow-up. Lung scan abnormalities are summarised in Table 1, 18 patients $(46 \%)$ had normal scans.

At follow-up, 43 patients $(93 \%)$ had chest $x$-ray film taken. Of these, 20 were normal (46\%) and in $10(23 \%)$ the only abnormalities were linear scars. The heart size was increased in 2 and the main pulmonary artery was enlarged (Fleischner, 1962) in 2 other patients. The width of the right descending pulmonary artery was normal in all.

In 5 patients, other chest $x$-ray abnormalities were seen: apical bullae were seen in 1 , calcified tuberculous lymph nodes in 1, evidence of a lobectomy many years before in another, and scoliosis in 2. The remainder of the abnormalities, relevant to pulmonary embolism, are summarised in Table 2. These patients were classified as without additional cardiorespiratory disease since these abnormalities were usually minor, often not recognised at initial diagnosis of embolism, and did not result in cardiorespiratory dysfunction. 
Table 1 Results of perfusion lung scans

\begin{tabular}{|c|c|c|c|c|c|c|}
\hline & $\begin{array}{l}\text { No. of } \\
\text { patients }\end{array}$ & Normal & $\begin{array}{l}\text { Single } \\
\text { defects }\end{array}$ & $\begin{array}{l}\text { Multiple } \\
\text { defects }\end{array}$ & $\begin{array}{l}\text { General decreased } \\
\text { perfusion one lung }\end{array}$ & $\begin{array}{l}\text { General decreased } \\
\text { perfusion one lung plus } \\
\text { defect other lung }\end{array}$ \\
\hline Without additional cardiorespiratory disease & 39 & $\begin{array}{l}18 \\
(46 \%)\end{array}$ & 7 & 7 & 5 & 2 \\
\hline With additional cardiorespiratory disease & 9 & $\begin{array}{c}2 \\
(22 \%)\end{array}$ & 1 & 5 & 1 & 0 \\
\hline All patients & 48 & $(42 \%)$ & 8 & 12 & 6 & 2 \\
\hline
\end{tabular}

Table 2 Results of chest $\mathrm{x}$-ray examination

\begin{tabular}{|c|c|c|c|c|c|c|}
\hline & No. of patients & Normal & Oligaemia & Scars & Pleural shadows & Raised diaphragm \\
\hline Without additional cardiorespiratory disease & 43 & $\begin{array}{l}20 \\
(46 \%)\end{array}$ & 1 & $17 \star$ & 8 & 3 \\
\hline $\begin{array}{l}\text { With additional cardiorespiratory disease } \\
\text { All patients }\end{array}$ & $\begin{array}{r}9 \\
52\end{array}$ & $\begin{array}{c}0 \\
20 \\
(38 \%)\end{array}$ & $\begin{array}{l}5 \\
6\end{array}$ & 26 & $\begin{array}{r}2 \\
10\end{array}$ & $\begin{array}{l}1 \\
4\end{array}$ \\
\hline
\end{tabular}

*Only abnormality in 10.

tAll patients had other abnormality.

\section{PATIENT WITH ADDITIONAL}

\section{CARDIORESPIRATORY DISEASE}

Of the 10 patients seen at follow-up and who initially had additional cardiorespiratory disease, 4 were asymptomatic. Five had varying degrees of exertional dyspnoea, and 2 of these patients had chronic bronchitis with occasional acute exacerbations. Two patients had had episodes suggestive of recurrent pulmonary embolism and infarction with pleuritic chest pain and haemoptysis. These occurred 11 and 46 months after the initial embolic episode.

Only 2 of these patients were free of abnormal physical signs; 4 had a double apical impulse (or fourth heart sound), 3 had systemic hypertension, and 2 had expiratory wheeze. One patient had mitral stenosis with atrial fibrillation, while another had a peripheral arteriovenous fistula in the arm and a hyperdynamic circulation.

The electrocardiograms were normal in 5 patients. Left ventricular abnormalities (left bundle-branch block or ST-T changes) were present in 3, and 1 showed an old inferior myocardial infarct. In the patient with mitral stenosis the electrocardiogram showed atrial fibrillation and right ventricular hypertrophy.

Nine patients had a perfusion lung scan and this was normal in 2 patients (22\%). The types and number of abnormalities seen are summarised in Table 1 . The same 9 patients had chest $x$-ray films. All the radiographs were abnormal, and in no patient were scars the only defects. The heart size was increased in 4 . The main pulmonary artery was enlarged in 1 patient with obstructive airways disease. The right descending pulmonary artery was abnormally large $(17 \mathrm{~mm})$ in another patient who had had a previous myocardial infarction, but was of normal size in the remaining patients. Chest $x$-ray films also showed evidence of old tuberculous infection and emphysema in 1 patient, emphysema alone in another, left atrial enlargement and upper lobe blood diversion in the patient with mitral stenosis, and upper lobe blood diversion in a patient with severe ventricular dysfunction from coronary artery disease. The abnormalities relating to pulmonary embolism are summarised in Table 2.

\section{LATE CARDIAC \\ CATHETERISATION STUDIES}

Fourteen patients had right heart catheterisation and pulmonary arteriography 2 to 72 months after initial treatment. Of 9 patients without additional cardiorespiratory disease, only 1 had an abnormal pulmonary artery pressure (described above). Of 5 patients with additional disease, 2 had raised pulmonary artery pressures, 1 as the result of mitral stenosis, the other because of left ventricular disease.

Pulmonary arteriograms were entirely normal in 5 patients (including the 4 restudied more than 6 months after the acute event) and only minor abnormalities were seen in the other 9 patients studied earlier. Pulmonary arteriograms were 
scored $^{1}$ using the method described by Miller et al. (1971), and the results are shown in Fig. 2. Of the 14 patients recatheterised, 6 were patients who had shown little arteriographic improvement when restudied immediately after the initial treatment period.

\section{Discussion}

These results indicate a satisfactory long-term prognosis for patients surviving the initial episode of acute massive pulmonary embolism and who have been treated by pulmonary embolectomy, streptokinase, or heparin. This favourable longterm outcome contrasts with the immediate high mortality of massive pulmonary embolism. The patients described in this series were a selected group as they had survived long enough after the immediate impact of massive embolism to be transferred from one hospital to another.

\section{LATE MORTALITY}

The late mortality of patients with additional cardiopulmonary disease $(41 \%, 7$ of 17 patients) was higher than that of patients whose initial embolism was not associated with other cardiopulmonary disease $(9 \%, 5$ of 55 patients). Though the number of patients with additional disease is small, this difference in mortality is striking. It has been postulated (Murphy and Bulloch, 1968) that late deaths attributable to non-resolution or recurrence of pulmonary embolism may be more common in

\begin{abstract}
${ }^{1}$ Severity of embolism is assessed in terms of both involvement of the main pulmonary arteries by clot and reduction in peripheral vessel perfusion. Reduction of perfusion is scored out of 18 and involvement of the pulmonary arteries by clot is scored out of 16 . The scores for perfusion and involvement are added together to give a final score. Thus a normal arteriogram scores 0 , while a final score of more than 17 indicates massive embolism.
\end{abstract}

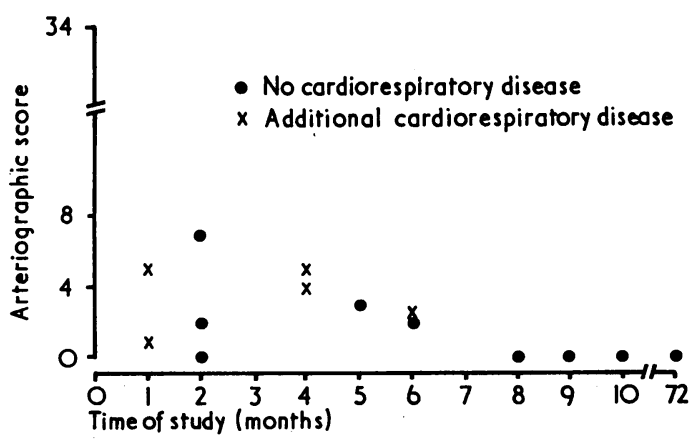

Fig. 2 Late pulmonary arteriographic results. patients with additional cardiopulmonary disease. However, we did not find any evidence of an increased incidence of non-resolution or recurrence in these patients; the high mortality in these patients resulted from progression of the additional cardiopulmonary disease.

There was no definite evidence of late death resulting solely from new or unresolved pulmonary embolism in any patient. One patient dying of chronic left ventricular disease was found to have both old and new pulmonary infarcts at necropsy. This is known to be a common finding in patients dying from cardiac failure. There was one late, sudden death in each group of patients, the cause of which was not confirmed by necropsy. Though pulmonary embolism cannot be excluded as the cause of death in these 2 patients, the clinical circumstances in 1 case suggested myocardial infarction, and the other patient had had a myocardial infarct 4 years before.

The high incidence of venous thromboembolism in patients with neoplastic disease is well known, and this series emphasises this association. It is of interest that of the 6 patients dying 3 months to 7 years later, 3 were not known to have malignant disease when they presented with massive embolism. It is possible that occult neoplasia was an unknown, but important, predisposing factor to embolism in these patients.

There was nothing to suggest that the form of initial treatment (pulmonary embolectomy, streptokinase, or heparin) was related to the nature or incidence of late deaths.

\section{RECURRENCE OF EMBOLISM}

It has been thought that there is a high incidence of recurrence of embolism in treated and untreated patients. The Urokinase Pulmonary Embolism Trial (1973) showed an incidence of 15 to 19 per cent recurrent embolism within the first 2 weeks of beginning treatment with urokinase or heparin. This has not been our experience. During the initial hospital treatment period, only 2 of these patients $(2 \%)$ had evidence of recurrence: 1 of these was the only patient in the entire series to have an operation on the inferior vena cava. Both these patients with early recurrence of embolism died during their initial admission, representing 13 per cent of all the hospital deaths in this series.

Although maintenance anticoagulants were seldom used, the incidence of clinically apparent recurrent pulmonary embolism over the period of follow-up was low. Three patients $(18 \%)$ with additional cardiorespiratory disease had evidence of recurrence: 1 patient who died of left ventricular disease had small fresh pulmonary infarcts at 
necropsy, and 2 other patients had a history of pleurisy and haemoptysis 1 and 4 years after initial treatment. None of these patients was on anticoagulants at the time of recurrence. Only 1 patient without additional cardiorespiratory disease had symptoms suggestive of recurrence. This low incidence of recurrence may have been the result of the vigorous initial treatment causing rapid restoration of the pulmonary circulation towards normal, and consequently an early return of systemic perfusion towards normal, decreasing the chance of further venous thrombosis. Other series (Crane et al., 1969) in which the patients were treated by heparin together with surgical procedure to the inferior vena cava or leg veins have shown a higher incidence of recurrent embolism, but there is little doubt that the majority of patients treated by embolectomy or thrombolytic drugs have more rapid restoration of the pulmonary circulation towards normal (Hirsh et al., 1968; Miller et al., 1971; Urokinase Pulmonary Embolism Trial, 1973). It is possible that patients with additional cardiorespiratory disease are more liable to recurrence of pulmonary embolism than those without, but the small number of such patients in this series does not permit comment on this aspect.

\section{RESOLUTION OF PULMONARY EMBOLISM}

This study does not attempt to assess the rate of resolution of treated pulmonary embolism. Comparative rates of early angiographic resolution produced by thrombolytic agents and heparin have been reported previously in other series (Urokinase Pulmonary Embolism Trial, 1973; Hirsh et al., 1968) and for some of the patients in the present series (Miller et al., 1971; Tibbutt et al., 1974). In this study, we have looked particularly for evidence of non-resolution 1 to 8 years after the initial embolic episode. We have found little such evidence other than the frequent persistent lung scan abnormalities. Similar findings have been reported by Paraskos et al. (1973).

There was no clinical evidence of pulmonary hypertension in any patient. The electrocardiogram usually reverted to normal, though some patients retained abnormal features which were part of the right ventricular 'strain' pattern present at the time of embolism. Only 2 patients had unexplained enlargement of the main pulmonary artery on chest $x$-ray film though in both the width of the right descending pulmonary artery was nurmal. It could be argued that the methods used for the detection of pulmonary hypertension in the majority of patients (clinical history and examination, electrocardiogram (Flowers and Horan, 1972), and chest $x$-ray) are insensitive and hence we failed to diagnose mild pulmonary hypertension in all cases where it existed. The results of late cardiac catheterisation suggest that this is not so. Fourteen patients were restudied and 11 had normal pulmonary artery pressures. In 2 of the 3 patients with pulmonary hypertension, this resulted from proven left heart disease. Only 1 patient had mild unexplained pulmonary hypertension. This patient had a normal pulmonary arteriogram and the raised pulmonary artery pressure may have resulted from small vessel obstruction caused by the previous embolism or from an unrelated cause. The results of arteriography are particularly significant since 6 of these patients were restudied because of poor angiographic resolution immediately after initial therapy and might be considered to be the patients most likely to develop subsequent pulmonary hypertension.

Thus, this study does not lend any support to the suggestion that massive pulmonary embolism leads to chronic thromboembolic pulmonary hypertension. It must be remembered, however, that all the patients were vigorously treated: our experience does not deny the possibility that untreated massive pulmonary embolism could lead ultimately to chronic thromboembolic pulmonary hypertension.

The perfusion lung scan, and in a small number the pulmonary arteriogram, were used to judge the completeness of resolution of pulmonary embolism. The lung scan is a sensitive method of detecting areas of abnormal lung perfusion, but the interpretation and standardisation of scans is difficult. Tow and Wagner (1967) described a group of patients with 'severe' pulmonary embolism (31 to $50 \%$ of the lung involved), only 1 of whom had a completely normal lung scan at 2 months. The Urokinase Pulmonary Embolism Trial (1973) showed that approximately 75 per cent of lung scans had resolved in patients treated with urokinase or heparin at 1 year: the majority of these patients did not have massive embolism.

We have not attempted to quantify lung scans, but our findings in patients seen 1 to 9 years later show normal scans in 42 per cent. The remainder had varying abnormalities usually not more than slight, and sometimes corresponding to a scar or pleural thickening visible on chest $x$-ray film, raised diaphragm, or cardiac enlargement. More patients with additional cardiorespiratory disease had abnormal lung scans $(76 \%)$ and chest $x$-ray films $(100 \%)$ than patients without additional disease in whom 54 per cent of lung scans and 54 per cent of chest $x$-ray films were abnormal. This is not unexpected since these lung scan abnormalities may result from the associated disease rather than from pulmonary embolic disease and do not necessarily 
reflect non-resolution. There is no clear relation between completeness of resolution and initial treatment method (embolectomy or streptokinase).

The persistence of lung scan and, on occasion, electrocardiographic abnormalities is of obvious clinical significance when assessing a possible recurrence in a patient with a past history of pulmonary embolism.

Frequent minor pulmonary arteriographic abnormalities were seen in patients restudied within 6 months of initial treatment (Fig. 2). It is likely that resolution is not complete in some patients by 6 months. The results of perfusion lung scanning in a larger number of patients at a later date suggest that minor perfusion abnormalities may often persist for long periods or perhaps indefinitely. Such minor incomplete resolution does not seem to be responsible for any serious disturbance in the patient. We do not know whether such incomplete resolution, if followed for several decades, might eventually lead to chronic thromboembolic pulmonary hypertension.

These patients were admitted under the care of Dr. R. V. Gibson, Dr. M. Honey, Dr. G. A. H. Miller, and Mr. M. Paneth. We thank them for their advice in the preparation of this paper.

\section{References}

Crane, C., Hartsuck, J., Birtch, A., Couch, N. P., Zollinger, R., Matloff, J., Dalen, J., and Dexter, L. (1969). The management of major pulmonary embolism. Surgery, Gynecology and Obstetrics, 128, 27-36.

Dalen, J. E., and Alpert, J. S. (1975). Natural history of pulmonary embolism. Progress in Cardiovascular Disease, 17, 259-270.

Fleischner, F. G. (1962). Pulmonary embolism. Clinical Radiology, 13, 169-182.

Flowers, N. C., and Horan, L. G. (1972). Subtle signs of right ventricular enlargement and their relative importance. In Advances in Electrocardiography, p. 297. Ed. by R. C. Schlant and J. W. Hurst. Grune and Stratton, New York.
Hirsh, J., Hale, G. S., McDonald, I. G., McCarthy, R. A., and Pitt, A. (1968). Streptokinase therapy in acute major pulmonary embolism: effectiveness and problems. British Medical fournal, 4, 729-734.

Magidson, O., and Jacobson, G. (1955). Thrombosis of the main pulmonary arteries. British Heart fournal, 17, 207-218.

Miller, G. A. H., Hall, R. J. C., and Paneth, M. (1977). Pulmonary embolectomy, heparin and streptokinase-their place in the treatment of acute massive pulmonary embolism. American Heart fournal, 93, 568-574.

Miller, G. A. H., Sutton, G. C., Kerr, I. H., Gibson, R. V., and Honey, M. (1971). Comparison of streptokinase and heparin in treatment of isolated acute massive pulmonary embolism. British Medical fournal, 2, 681-684.

Murphy, M. L., and Bulloch, R. T. (1968). Factors influencing the restoration of blood flow following pulmonary embolization as determined by angiography and scanning. Circulation, 38, 1116-1126.

Paneth, M. (1967). The treatment of pulmonary embolism. British fournal of Surgery, 54, 468-470.

Paraskos, J. A., Adelstein, S. J., Smith, R. E., Rickman, F. D., Grossman, W., Dexter, L., and Dalen, J. E. (1973). Late prognosis of acute pulmonary embolism. New England Fournal of Medicine, 289, 55-58.

Phear, D. (1960). Pulmonary embolism. A study of late prognosis. Lancet, 2, 832-835.

Tibbutt, D. A., Davies, J. A., Anderson, J. A., Fletcher, E. W. L., Hamill, J., Holt, J. M., Lea Thomas, M., De J. Lee, G., Miller, G. A. H., Sharp, A. A., and Sutton, G. C. (1974). Comparison by controlled clinical trial of streptokinase and heparin in treatment of life-threatening pulmonary embolism. British Medical fournal, 1, 343-347.

Tow, D. E., and Wagner, H. N. (1967). Recovery of pulmonary arterial blood flow in patients with pulmonary embolism. New England fournal of Medicine, 276, 1053-1059.

Urokinase Pulmonary Embolism Trial (1973). Circulation, 47-48, Suppl. 11.

Wood, P. (1956). Diseases of the Heart and Circulation, 2nd ed., p. 816. Eyre and Spotiswoode, London.

Requests for reprints to Dr. Roger Hall, Cardiac Department, Brompton Hospital, Fulham Road, London SW3 6HP. 